\title{
Epidemiological aspects of heart diseases (Review)
}

\author{
AIMIN SHI $^{1}$, ZIQI TAO ${ }^{2}$, PENG WEI $^{3}$ and JING ZHAO ${ }^{4}$ \\ ${ }^{1}$ School of Public Health of Nanjing Medical University, Nanjing, Jiangsu 211166; \\ Departments of ${ }^{2}$ Science and Education, ${ }^{3}$ Cardiology, and ${ }^{4}$ Science and Education Division, \\ Xuzhou Central Hospital, Xuzhou, Jiangsu 221009, P.R. China
}

Received December 31, 2015; Accepted July 25, 2016

DOI: 10.3892/etm.2016.3541

\begin{abstract}
Cardiovascular diseases (CVDs) are the leading cause of mortality worldwide. Coronary heart disease (CHD) is the main cause of mortality in heart patients following stroke, rheumatic heart disease and myocardial infarctions. Approximately $80 \%$ of individuals succumb to CVDs, due to poor living conditions in low and middle income families and malnutrition. Infectious diseases, human immunodeficiency, tuberculosis, malaria, high blood pressure or hypertension, obesity and overweight, and nutritional disorders including smoking, excessive alcohol consumption, high salt and sugar intake, as well as other factors are responsible for CVDs and CHDs in young as well as elderly individuals. The focus of the present review are recent epidemiological aspects of CVD and CHD as well as the usefulness of a Mediterranean diet for heart patients and the prevention of heart diseases.
\end{abstract}

\section{Contents}

1. Introduction

2. Risk factors

3. Epidemiological studies

4. Mediterranean diet for heart patients

5. Conclusion

\section{Introduction}

Chronic cardiovascular diseases (CVDs) are considered the leading cause of mortality worldwide $(1,2)$. Mortalities by CVDs occur mainly due to coronary heart disease (CHD), stroke, rheumatic heart, and myocardial infarction (MI). Globally approximately 17.5 million individuals succumb to various heart diseases. Approximately $80 \%$ of these

Correspondence to: Dr Ziqi Tao, Department of Science and Education, Xuzhou Central Hospital, 199 Jiefang South Road, Xuzhou, Jiangsu 221009, P.R. China

E-mail: uk092w@163.com

Key words: cardiovascular diseases, coronary heart disease, rheumatic heart disease deaths occur in low and middle income families and in poor countries (3). It is estimated by WHO that approximately 20 million individuals are likely to succumb to CVDs in the year 2015 (4). In addition, non-communicable diseases are to account for over $75 \%$ of mortalities worldwide (1). Infectious diseases, tuberculosis, and malaria followed by nutritional disorders are the primary cause of such mortalities (5). CVDs are also the largest contributor to global mortality of heart patients $(3,6)$. The epidemiology of heart diseases has been described by many researchers in the literature. For the epidemiological studies of heart diseases, a significant percentage of CVD mortalities occur in younger individuals and children in developing nations due to malnutrition, low income, poverty, unemployment, excessive alcohol consumption, tobacco smoking, car accidents, and other factors (5). Approximately $41 \%$ individuals die between the age of 35 and 64 years in South Africa, 35\% in India, 28\% in Brazil, $12 \%$ in the USA, and $9 \%$ in Portugal (7).

The median age of heart attack and first stroke and death from ischemic heart disease (IHD) as well as acute myocardial infarction (AMI) are very important (3). Hypertension, high blood pressure (HBP), high blood cholesterol, obesity, diabetes, reduced physical activity, psychological stress and other factors are also responsible for CVDs. The historical trends in CV mortality and tobacco use in North America increased between 1900 and 1990 (8). In the UK, a 38-year follow-up study of men showed that baseline differences in tobacco use, HBP pressure, and cholesterol were associated with a 10- to 15-year shorter life expectancy (9). The rise in cigarette smoking in young individuals indicates increased tobacco-related mortalities in many countries (10). By the year 2030, WHO (2008) projects that over $80 \%$ of tobacco-related deaths are to occur in young people in developing countries. Currently, malnutrition and obesity occur within the same population in many low and middle income families $(11,12)$. Malnutrition is common in many low and middle income countries of Africa, Latin America, and South Asia (7). Approximately 300 million individuals suffer from obesity (13). The global prevalence of overweight in children aged 5-17 years is 10\%, varying from under 2\% in Sub-Saharan Africa to over 30\% in the USA (14). The epidemiological research of CVDs suggests that dietary changes are associated with nutritional transition, specifically the increasing consumption of energy-dense diets high in fats, oils, sodium, and sugars, which contribute to an increase in CVD incidence in low and middle income families (15). 


\section{Mediterranean diet for heart patients}

The kilo caloric intake from selected food groups in China, India, Mexico, Egypt, and South Africa from 1980 to 1982 were compared to the kilo caloric intake in 2001 to 2003 using data from the Food and Agriculture Organization of the United Nations Statistical Database (FAOSTAT) (16). In China, total LCD (low-calorie diet) increased from 2,327 to 2,940 kcal, and meat consumption increased by over $246 \%$ from 1980 to 2003. There was also a significant increase in the intake of oils in China, with three types of oils increasing over $100 \%$ : palm oil $(+640 \%)$, soybean oil $(+635 \%)$, and vegetable oils $(+259 \%)$. On the positive side, the intake of fruits and vegetables also increased by 600 and 367\%, respectively (16). Between 1980 and 2003 , there has been an increase in meat as well as greasy food consumption in China, as well as increased use of tropical oils (which is associated with high cholesterol and not healthy for heart) in preparing food. Similarly, in India, Mexico, South Africa and other nations the elevated kcal intake of palm oil in the diets was reported (16). The causes of the decline in CVDs in developed countries offer potential lessons for achieving similar results in developing countries. The major risk factors for CVDs have been reported for childhood and adolescence (17-20). These include use of tobacco, diets and physical activities, overweight and obesity, and adverse childhood experiences. Poor social life circumstances in childhood have also been linked to CVDs later in life in different cohort studies conducted in the USA and Europe $(21,22)$.

A cohort study of 20,040 individuals was conducted over 11 years to determine the risk of stroke incidence. Four measures of health were examined: smoking, low physical activity, low plasma vitamin $\mathrm{C}$ levels (used as a proxy for fruit and vegetable intake), and not drinking alcohol in moderation (abstaining from alcohol or consuming more than 14 drinks per week) and the results indicated that one or more of the abovementioned risk factors alone or in combination with other risk factors constituted a $>2$-fold increase in stroke incidence (23). This is consistent with prior findings in large cohort studies on men and women in the USA whereby a healthy diet and lifestyle of not smoking, regular exercise, moderate alcohol consumption, and not being overweight was associated with an almost $80 \%$ lower risk of IHD compared to having none of the abovementioned healthy lifestyle components (24). The major risk factors for CVD in young individuals or adults include fats, abnormal blood lipids, tobacco smoking, alcohol, abdominal obesity, psychosocial factors, hypertension, and diabetes. There is strong evidence that, excessive salt and sodium intake significantly increases CVD risk and that reduction in sodium intake decreases incidence of CVDs (25). A high-salt intake in diet increases blood pressure. In another study, three major dietary patterns were identified: i) Oriental (high intake of tofu and soya; ii) Western (high in fried foods, salty snacks, eggs, and meat); and iii) prudent (high in fruits and vegetables). The results showed the Western dietary pattern was associated with an increased risk of CHD in animals and humans globally, whereas the prudent pattern with fruits and vegetables was associated with a lower risk of heart diseases in children, young and adult individuals (26).

Increased daily consumption of alcohol has been reported as a great risk factor of CHD in individuals of all ages in low and middle income groups (27). The WHO estimates that the harmful use of alcohol was responsible for 3.8\% deaths and $4.5 \%$ of the global burden of heart diseases in 2004 (3). In the past few decades, consumption of alcohol has increased in countries undergoing nutritional transition, such as India and China, and Russia, where it contributes significantly to overall mortality among men (WHO Expert Committee, 2007) (2). Excessive alcohol intake is associated with increased risk for hypertension, stroke, coronary artery disease, and other forms of CVDs; however, evidence conducted in various populations suggests that light to moderate intake of alcohol may reduce the risk of CHD (2). The relationship between one drink per day for women and no more than two drinks per day for men constitute moderate drinking for health of heart (28). Physical exercise is also important for the health of heart as WHO and the Food and Agriculture Organization (FAO) suggest the importance of physical activity as a key determinant of obesity, CVD, and diabetes (29). Obesity or overweight issues, reported from developed and developing countries are considered risks of CVDs (30). Malnutrition remains highly prevalent compared to overweight and obesity, especially among women and children (31). The intake of cereals is also important in China, where $58 \%$ of total calorie intake is consumed compared to meat, $13 \%$, and cooking oils, $17 \%$ (32). The national data from the Office of National Statistics indicates that cereals and related products account for $31 \%$ of calories with other major categories including meat, $15 \%$; milk and related products, $19 \%$; and beverages, $10 \%$ (33).

In a study on HBP it was found that approximately $54 \%$ of individuals experience stroke, 47\% IHD, 75\% hypertensive disease and $25 \%$ other CVDs that were attributable to hypertension. Approximately 7.6 million individuals succumb to hypertension, with $13.5 \%$ of the total number of annual global deaths being attributable to HBP (34). It is estimated that the current age-standardized prevalence rate of hypertension is $17.7 \%$, which amounts to approximately 177 million individuals, while $20 \%$ of individuals in China may succumb to HBP due to a high intake of salt $(35,36)$. The Framingham study showed a connection between hypercholesterolemia and increased risk of CHD in 1960 with the finding that lower levels of high-density lipoprotein (HDL) cholesterol as well as elevated levels of low-density lipoprotein (LDL) cholesterol were associated with increased risk of CHD $(37,38)$. Elevated triglycerides levels also increase the risk of CVD $(39,40)$. The results of several randomized controlled trial studies have shown that the reduction of LDL cholesterol, both in primary and secondary prevention, is associated with a lower incidence of CHDs (41-43). The INTERHEART study found that abnormal blood lipids were the most important risk factor for MI, by odds ratio in all global regions (44). Elevated cholesterol levels were the third leading risk factor for the worldwide mortality of young and elderly individuals, after hypertension and smoking (45).

The risk of CVD associated with diabetes is even greater in women and younger individuals. Having diabetes may be equivalent to aging by at least 15 years with regard to the clinical manifestations of CVD $(46,47)$. Diabetes is associated with serious health consequences and is a major risk factor for $\mathrm{CHD}$ and stroke. Depression and depressive symptoms are also associated with behaviors that increase CVD risk (48). Anxiety, 
anger, hostility, chronic and acute stress, and lack of social support are factors associated with increased CVD morbidity and mortality. The acute stress from traumatic injury, death of a relative, earthquakes, terrorist attacks or other factors have been associated with significant temporal increases in the incidence of MI in young or adults (49). Research has also shown that air pollution and respiratory problems have adverse health outcomes associated with lung-heart or cardiovascular effects (50). The burning of coal and wood used for cooking is also a significant contributor of air pollution in developing countries. Excessive smoke inhalation may cause cancer and heart attacks in humans. The epidemiological studies of heart diseases have also shown that long-term exposure to smoke can increase the risk of CVD (51).

\section{Epidemiological studies}

Molecular genetics and advanced statistical methods, used for the study of heart diseases and proteomics methods can lead to potential biomarkers that can identify risk of CVDs, which in turn, can improve the prediction of acute cardiovascular events (52). Pharmacogenetics can be useful in the development of more effective medications, and the management of CVD in children, young and adult population. Rheumatic heart disease (RHD), Chagas disease, and infectious pericarditis and cardiomyopathies continue to cause a substantial burden of disease and death in some low and middle income countries (53). A better epidemiological understanding of infectious forms of CVD in developing countries, and efforts to improve health care are needed to prevent and treat these diseases. Infectious diseases such as HIV/AIDS, and tuberculosis (TB), and other diseases such as malaria, typhoid and diabetes are also important for their association with CVD (53). In order to study CVDs in the developing and developed nations, efficient epidemiological programs should be designed.

Epidemiological studies of CHD have been described (54) for preventive cardiology. CVDs cause approximately one-third of mortalities worldwide (55). CHD accounts for the maximum proportion of CVDs for which risk factors are hypertension, smoking, diabetes, high cholesterol, higher level of LDL, obesity and overweight causing death of heart patients. Assessment of short- or long-term risk prediction algorithms is useful to identify patients that can benefit most from risk factor interventions. The evaluation of new risk factors and screening for subclinical atherosclerosis are imperative in the identification of individuals that are at the highest risk of CVD. The prevention of CHDs must focus on identifying and managing risk factors in individual cases as well as the population through primordial, primary, and secondary prevention. Epidemiological studies have provided a hypothesis based on clinical trials for CHD and the efficacy of risk-factor interventions, which are the basis of prevention of heart diseases (55). Further investigations on screening and intervention strategies is important to determine the effect on the prevention of CHDs and CVDs.

Recent advances in the epidemiology, pathogenesis and prognosis of acute heart failure and cardiomyopathy have been described from Africa (56). A large prospective registry showed that acute decompensated heart failure is caused by hypertension, cardiomyopathy and RHD in $90 \%$ of cases. This is a pattern that is in contrast with the dominance of coronary artery disease in North America and Europe (56). Acute heart failure is a disease of young individuals with a mean age of 52 years and occurs equally in men and women. This observation was associated with high mortality at 6 months (approximately $18 \%$ ), but was similar to that observed in non-African heart failure registries, suggesting that heart failure was a dire prognosis globally, regardless of the aetiology. Investigations into the molecular genetics of dilated cardiomyopathy, hypertrophic cardiomyopathy and arrhythmogenic right ventricular cardiomyopathy in populations in Africa are consistent with observations elsewhere (56). In addition, the study of genotypephenotype correlations in a large number of cardiomyopathy individuals is due to the same mutation (56). Thus the use of proven treatments, and the control of hypertension in various populations should be increased.

\section{Mediterranean diet for heart patients}

The American Heart Association (AHA) has recommended following a Mediterranean diet for heart patients (57), in which more fruits and vegetables are included. At least 16 countries border the Mediterranean Sea and their diets vary among these countries. There are numerous differences in culture, ethnic background, religion, economy and agricultural production that result in a difference in Mediterranean diets. The common Mediterranean dietary pattern has these characteristics: high consumption of fruits, vegetables, bread and other cereals, potatoes, beans, nuts and seeds, olive oil which is an important source of monounsaturated fats, dairy products, fish and poultry consumed in low to moderate amounts, and a modest amount of red meat (57). In such a diet, eggs are consumed 0-4 times a week and wine in low-to-moderate amounts. Individuals who adhere to an average Mediterranean diet usually eat less saturated fats than those who consume an average American diet. Saturated fat consumption is well within dietary guidelines. It is known that over $50 \%$ of fat calories in a Mediterranean diet are derived from monounsaturated fats, mainly from olive oil. Monounsaturated fats do not raise the level of blood cholesterol in the same way as saturated fat. The incidence of heart disease in Mediterranean countries is lower than that in the United States, as are mortality rates. Diet as well as lifestyle factors, such as more physical activity and extended social support systems may also be important in heart diseases. In recent advances in preventive cardiology and lifestyle medicine, public policy approaches to the prevention of heart disease and stroke have been described (58). The global epidemiology of heart disease and stroke in the 20 th century showed marked variations in cause-specific mortality rates between developed and developing countries due to trends of social and political origins of cardiovascular risk. Thus, governments and the private sectors are attempting to educate the public and coordinate public efforts to retard the global epidemic of heart disease and stroke (59). The Osaka Declaration has emphasized factors for the health sector that serve as impediments to heart health (60).

Success in the treatment of heart diseases and conditions of MI, valvular heart disease, and arrhythmias has improved patient survival. In the elderly, congestive heart failure is the leading cause for admission to hospitals in the United 
States, which is extremely expensive for health care (61). African-Americans, aged 45-65 years, have a 70\% higher rate of heart failure as shown in the Census Bureau and the Centers for Disease Control and Prevention, with a mortality rate 2.5 -fold higher than that of the Caucasian population. The Hispanic/Latino population has a higher incidence of heart failure than the Caucasian non-Hispanic population. However, more population-based studies are needed to estimate the prevalence of heart failure in the Hispanic population (62).

Useful sites for heart disease research include: The American College of Cardiology (http://www.acc.org/), for up to date heart failure patient's information (http://www. uptodate.com/home); National Library of Medicine, (https:// www.nlm.nih.gov/); National Heart, Lung, and Blood Institute (http://www.nhlbi.nih.gov/); European Society of Cardiology (http://www.escardio.org/); and Heart Failure Online, http:// www.heartfailure.org/.

Recent investigations on CVDs in Europe for 2014 have shown that, epidemiological aspects have been updated (63). CVDs and in particular CHD and stroke have been previously demonstrated in European countries (63). CVDs cause more deaths among Europeans than other countries, and cause more than twice as many deaths as cancer. It has also been reported that for some Eastern European countries, including Russia and the Ukraine, the mortality rate for CHD in 55- to 60-year-old individuals is greater than the equivalent rate in France. The epidemiology of CVD in the UK in 2014 was described with regard to mortality, prevalence and treatment costs (64). In the year 2012, CVD was the most common cause of mortality in the UK for women at $28 \%$ of all female deaths, but not for men, whereas cancer is currently the most common cause of mortality at $32 \%$ of all male deaths. Mortality from CVD varies widely throughout the UK, with the highest age-standardized CVD death rates in Scotland $(347 / 100,000)$ and the North of England (320/100,000 in the North West). The prevalence of CHDs is also highest in the North of England (4.5\% in the North East) and Scotland (4.3\%). Approximately 3 times more men have had an MI as compared to women. Thus, primary and secondary prevention measures are important to reduce the burden of CVD and inequalities in CVD mortality rate and prevalence. The epidemiology of heart failure has been reported and it has been suggested that CHD is prevalent (65). The excessive use of tobacco smoking, alcohol consumption and other factors are also responsible for heart diseases.

\section{Conclusion}

The present review focused on recent epidemiological aspects of CVD and CHD as well as the benefits of a Mediterranean diet for heart patients and its usefulness in preventing cardiovascular disease. In general, there are several risk factors such as high blood cholesterol, high blood pressure, obesity and family history that are associated with CVD and CHD. Some of these factors can be controlled by a good diet. Results obtained from several studies support the positive effects of the Mediterranean diet for the primary prevention of cardiovascular disease. The Mediterranean diet is characterized by a high intake of olive oil, fruit, vegetables, and cereals; a moderate intake of fish and poultry, and a low intake of dairy products, red meat, processed meats, and sweets, as well as wine in moderation, consumed with meals. Future studies may shed more light on the notable effects of the Mediterranean diet on the prevention of cardiovascular disease.

\section{References}

1. Adeyi O, Smith O and Robles S: Public policy and the challenge of chronic noncommunicable diseases. The World Bank, Washington, DC, 2007.

2. WHO Expert Committee on Problems Related To Alcohol Consumption: Second Report. World Health Organization Technical Report Series. World Health Organization, Geneva, pp1-65, 2007. Mongolian STEPS Survey on the Prevalence of Noncommunicable Disease Risk Factors 2006. World Health Organization Western Pacific Region, Manila, Philippines, 2007.

3. World Health Statistics 2009. WHOSIS (World Health Organization Statistical Information System). World Health Organization, Geneva, pp1-149, 2009.

4. The Current Evidence For The Burden Of Group A Streptococcal Diseases. Discussion Papers On Child Health. World Health Organization, Department of Child and Adolescent Health and Development, Geneva, pp1-60, 2005.

5. Beaglehole R and Bonita R: Global public health: a scorecard. Lancet 372: 1988-1996, 2008

6. Fuster V and Kelly BB (eds): Epidemiology of cardiovascular disease. In: Promoting Cardiovascular Health in the Developing World: A Critical Challenge to Achieve Global Health. National Academies Press, Washington, DC, 2015.

7. Leeder S, Raymond S, Greenberg H, Liu H and Esson K: A Race Against Time: The Challenge Of Cardiovascular Disease In Developing Economics. Columbia University, New York, NY, ppl-54, 2004

8. Shopland DR: Tobacco use and its contribution to early cancer mortality with a special emphasis on cigarette smoking. Environ Health Perspect 103 (Suppl 8): 131-142, 1995.

9. Clarke R, Emberson J, Fletcher A, Breeze E, Marmot M and Shipley MJ: Life expectancy in relation to cardiovascular risk factors: 38 year follow-up of 19,000 men in the Whitehall study. BMJ 339: b3513, 2009.

10. Shafey O, Eriksen M, Ross H and Mackay J: The tobacco atlas. 3rd edition. American Cancer Society, Atlanta, 2009.

11. WHO: Childhood overweight and obesity. http://www.who. int/dietphysicalactivity/childhood/en/WHO. Accessed December 12, 2008. The Global Burden Of Disease: 2004 Update. World Health Organization Geneva, 2008. Who Report On The Global Tobacco Epidemic, 2008: The Mpower Package. World Health Organization, Geneva, 2008.

12. Reddy SP, Panday S, Swart D, Jinabhai CC, Amosun SL, James S, Monyeki KD, Stevens G, Morejele N, Kambaran NS, et al: Umthenthe uhlaba usamila: the South African Youth Risk Behavior Survey 2002. South African Medical Research Council, Cape Town, 2003.

13. Misra A and Khurana L: Obesity and the metabolic syndrome in developing countries. J Clin Endocrinol Metab 93 (Suppl 1): S9-S30, 2008.

14. Bhardwaj S, Misra A, Khurana L, Gulati S, Shah P and Vikram NK: Childhood obesity in Asian Indians: a burgeoning cause of insulin resistance, diabetes and sub-clinical inflammation. Asia Pac J Clin Nutr 17 (Suppl 1): 172-175, 2008.

15. Hu FB: Globalization of food patterns and cardiovascular disease risk. Circulation 118: 1913-1914, 2008.

16. Khan M and Mensah G: Changing practices to improve dietary outcomes and reduce cardiovascular risk: a food company's perspective. Background Paper Commissioned by the Committee, Purchase, NY, 2009.

17. Barker DJ, Martyn CN, Osmond C, Hales $\mathrm{CN}$ and Fall $\mathrm{CH}$ : Growth in utero and serum cholesterol concentrations in adult life. BMJ 307: 1524-1527, 1993.

18. Celermajer DS and Ayer JG: Childhood risk factors for adult cardiovascular disease and primary prevention in childhood. Heart 92: 1701-1706, 2006.

19. Freedman DS, Khan LK, Dietz WH, Srinivasan SR and Berenson GS: Relationship of childhood obesity to coronary heart disease risk factors in adulthood: the Bogalusa Heart Study. Pediatrics 108: 712-718, 2001. 
20. Strong JP, Malcom GT, McMahan CA, Tracy RE, Newman WP III, Herderick EE and Cornhill JF: Prevalence and extent of atherosclerosis in adolescents and young adults: implications for prevention from the Pathobiological Determinants of Atherosclerosis in Youth Study. JAMA 281: 727-735, 1999.

21. Smith GD, McCarron P, Okasha M and McEwen J: Social circumstances in childhood and cardiovascular disease mortality: prospective observational study of Glasgow University students. J Epidemiol Community Health 55: 340-341, 2001.

22. Galobardes B, Smith GD and Lynch JW: Systematic review of the influence of childhood socioeconomic circumstances on risk for cardiovascular disease in adulthood. Ann Epidemiol 16 91-104, 2006

23. Myint PK, Luben RN, Wareham NJ, Bingham SA and Khaw KT: Combined effect of health behaviours and risk of first ever stroke in 20,040 men and women over 11 years' follow-up in Norfolk cohort of European Prospective Investigation of Cancer (EPIC Norfolk): Prospective population study. BMJ 338: b349, 2009.

24. Chiuve SE, Rexrode KM, Spiegelman D, Logroscino G, Manson JE and Rimm EB: Primary prevention of stroke by healthy lifestyle. Circulation 118: 947-954, 2008.

25. He FJ and MacGregor GA: A comprehensive review on salt and health and current experience of worldwide salt reduction programmes. J Hum Hypertens 23: 363-384, 2009.

26. Iqbal $R$, Anand S, Ounpuu S, Islam S, Zhang X, Rangarajan S, Chifamb J, Al-Hinai A, Keltai M and Yusuf S; INTERHEART Study Investigators: Dietary patterns and the risk of acute myocardial infarction in 52 countries: results of the INTERHEART study. Circulation 118: 1929-1937, 2008.

27. Rehm J, Mathers C, Popova S, Thavorncharoensap M, Teerawattananon $\mathrm{Y}$ and Patra $\mathrm{J}$ : Global burden of disease and injury and economic cost attributable to alcohol use and alcohol-use disorders. Lancet 373: 2223-2233, 2009.

28. Mukamal KJ, Chiuve SE, Rimm EB, Mukamal KJ, Chiuve SE and Rimm EB: Alcohol consumption and risk for coronary heart disease in men with healthy lifestyles. Arch Intern Med 166 : 2145-2150, 2006

29. Diet, Nutrition And The Prevention Of Chronic Diseases: Report Of A Joint Who/Fao Expert Consultation. Who Technical Report Series. World Health Organization, Geneva, 2003.

30. Sassi F, Cecchini M, Lauer J and Chisholm D: Improving Lifestyles, Tackling Obesity: The Health And Economic Impact Of Prevention Strategies. Organisation for Economic Co-operation and Development, Paris, 2009.

31. Caballero B: A nutrition paradox-underweight and obesity in developing countries. N Engl J Med 352: 1514-1516, 2005.

32. Wang L, Qi X, Chen C, Ma J, Li L, Rao K, Kong L, Ma G, Xiang H, $\mathrm{Pu}$ J, et al (eds). Survey and report on the status of nutrition and health of the Chinese people: a comprehensive report for 2002 People's Medical Publishing House, Beijing, 2005.

33. Office of the National Statistics, Report, 2003.

34. Lawes CMM, Vander Hoorn S and Rodgers A; International Society of Hypertension: Global burden of blood-pressure-related disease, 2001. Lancet 371: 1513-1518, 2008.

35. He J, Gu D, Chen J, Wu X, Kelly TN, Huang JF, Chen JC, Chen CS, Bazzano LA, Reynolds K, et al: Premature deaths attributable to blood pressure in China: a prospective cohort study. Lancet 374: 1765-1772, 2009.

36. Yang G, Kong L, Zhao W, Wan X, Zhai Y, Chen LC and Koplan JP: Emergence of chronic non-communicable diseases in China. Lancet 372: 1697-1705, 2008

37. Kannel WB, Dawber TR, Kagan A, Revotskie N and Stokes J II: Factors of risk in the development of coronary heart diseasesix year follow-up experience. The Framingham Study. Ann Intern Med 55: 33-50, 1961

38. Kannel WB, Castelli WP, Gordon T and McNamara PM: Serum cholesterol, lipoproteins, and the risk of coronary heart disease. The Framingham study. Ann Intern Med 74: 1-12, 1971.

39. Gotto AM Jr: Evolving concepts of dyslipidemia, atherosclerosis, and cardiovascular disease: the Louis F. Bishop lecture. J Am Coll Cardiol 46: 1219-1224, 2005

40. Manninen V, Tenkanen L, Koskinen P, Huttunen JK, Mänttäri M, Heinonen OP and Frick MH: Joint effects of serum triglyceride and LDL cholesterol and HDL cholesterol concentrations on coronary heart disease risk in the Helsinki Heart Study. Implications for treatment. Circulation 85: 37-45, 1992.
41. Downs JR, Clearfield M, Weis S, Whitney E, Shapiro DR, Beere PA, Langendorfer A, Stein EA, Kruyer W and Gotto AM Jr; for the AFCAPS/TexCAPS Research Group: Primary prevention of acute coronary events with lovastatin in men and women with average cholesterol levels: results of AFCAPS/TexCAPS Air Force/Texas Coronary Atherosclerosis Prevention Study. JAMA 279: 1615-1622, 1998

42. Sacks FM, Pfeffer MA, Moye LA, Rouleau JL, Rutherford JD, Cole TG, Brown L, Warnica JW, Arnold JM, Wun CC, et al: The effect of pravastatin on coronary events after myocardial infarction in patients with average cholesterol levels. Cholesterol and Recurrent Events Trial investigators. N Engl J Med 335: 1001-1009, 1996

43. Shepherd J, Cobbe SM, Ford I, Isles CG, Lorimer AR, MacFarlane PW, McKillop JH and Packard CJ; West of Scotland Coronary Prevention Study Group: Prevention of coronary heart disease with pravastatin in men with hypercholesterolemia. N Engl J Med 333: 1301-1307, 1995.

44. Yusuf S, Hawken S, Ounpuu S, Dans T, Avezum A, Lanas F, McQueen M, Budaj A, Pais P, Varigos J, et al; INTERHEART Study Investigators: Effect of potentially modifiable risk factors associated with myocardial infarction in 52 countries (the INTERHEART study): case-control study. Lancet 364: 937-952, 2004.

45. Lopez AD, Mathers CD, Ezzati M, Jamison DT and Murray CJL: Global burden of disease and risk factors. World Bank, Washington, DC, 2006.

46. Booth GL, Kapral MK, Fung K and Tu JV: Relation between age and cardiovascular disease in men and women with diabetes compared with non-diabetic people: a population-based retrospective cohort study. Lancet 368: 29-36, 2006b.

47. Huxley R, Barzi F and Woodward M: Excess risk of fatal coronary heart disease associated with diabetes in men and women: meta-analysis of 37 prospective cohort studies. BMJ 332: 73-78, 2006

48. Lichtman JH, Bigger JT Jr, Blumenthal JA, Frasure-Smith N, Kaufmann PG, Lespérance F, Mark DB, Sheps DS, Taylor $\mathrm{CB}$ and Froelicher ES; American Heart Association Prevention Committee of the Council on Cardiovascular Nursing; American Heart Association Council on Clinical Cardiology; American Heart Association Council on Epidemiology and Prevention; American Heart Association Interdisciplinary Council on Quality of Care and Outcomes Research; American Psychiatric Association: Depression and coronary heart disease: recommendations for screening, referral, and treatment: a science advisory from the American Heart Association Prevention Committee of the Council on Cardiovascular Nursing, Council on Clinical Cardiology, Council on Epidemiology and Prevention, and Interdisciplinary Council on Quality of Care and Outcomes Research: endorsed by the American Psychiatric Association. Circulation 118: $1768-1775,2008$

49. Figueredo VM: The time has come for physicians to take notice: the impact of psychosocial stressors on the heart. Am J Med 122: 704-712, 2009.

50. Brook RD: Cardiovascular effects of air pollution. Clin Sci (Lond) 115: 175-187, 2008.

51. Pope CA III, Burnett RT, Thurston GD, Thun MJ, Calle EE, Krewski D and Godleski JJ: Cardiovascular mortality and long-term exposure to particulate air pollution: epidemiological evidence of general pathophysiological pathways of disease. Circulation 109: 71-77, 2004.

52. Arnett DK, Baird AE, Barkley RA, Basson CT, Boerwinkle E, Ganesh SK, Herrington DM, Hong Y, Jaquish C, McDermott DA, et al; American Heart Association Council on Epidemiology and Prevention; American Heart Association Stroke Council; Functional Genomics and Translational Biology Interdisciplinary Working Group: Relevance of genetics and genomics for prevention and treatment of cardiovascular disease: a scientific statement from the American Heart Association Council on Epidemiology and Prevention, the Stroke Council, and the Functional Genomics and Translational Biology Interdisciplinary Working Group. Circulation 115: 2878-2901, 2007.

53. Gajalakshmi V, Peto R, Kanaka TS and Jha P: Smoking and mortality from tuberculosis and other diseases in India: retrospective study of 43000 adult male deaths and 35000 controls. Lancet 362: 507-515, 2003a.

54. Wong ND: Epidemiological studies of CHD and the evolution of preventive cardiology. Nat Rev Cardiol. 11: 276-289, 2014. 
55. Rosamond W, Flegal K, Furie K, Go A, Greenlund K, Haase N, Hailpern SM, Ho M, Howard V, Kissela B, et al; American Heart Association Statistics Committee and Stroke Statistics Subcommittee: Heart disease and stroke statistics-2008 update: a report from the American Heart Association Statistics Committee and Stroke Statistics Subcommittee. Circulation 117: e25-e146, 2008.

56. Sliwa K and Mayosi BM: Recent advances in the epidemiology, pathogenesis and prognosis of acute heart failure and cardiomyopathy in Africa. Heart 99: 1317-1322, 2013.

57. Mediterranean Diet. American Heart Association, Updated: August 11, 2014

58. Pearson TA: Recent Advances in Preventive Cardiology and Lifestyle Medicine. Public Policy Approaches to the Prevention of Heart Disease and Stroke. Circulation 124: 2560-2571, 2011.

59. The Victoria Declaration On Heart Health Victoria. Health and Welfare Canada, Victoria, Canada, 1992.

60. The Osaka Declaration: Health, Economics, And Political Action: Stemming The Global Tide Of Cardiovascular Disease. Declaration of the Fourth International Heart Health Conference, Osaka (Japan), May 2001. Health Canada, 2001.
61. Elixhauser A, Yu K, Steiner C and Bierman AS: Most Common reasons for hospitalizations by age groups. In: Hospitalization in the United States, 1997. HCUP Fact Book No. 1. Agency for Healthcare Research and Quality, Rockville, MD, p13, 2000.

62. Bahrami H, Kronmal R, Bluemke DA, Olson J, Shea S, Liu K, Burke GL and Lima JA: Differences in the incidence of congestive heart failure by ethnicity: the multi-ethnic study of atherosclerosis. Arch Intern Med 168: 2138-2145, 2008.

63. Nichols M, Townsend N, Scarborough P and Rayner M Cardiovascular disease in Europe 2014: epidemiological update. Eur Heart J 35: 2950-2959, 2014.

64. Bhatnagar P, Wickramasinghe K, Williams J, Rayner M and Townsend N: The epidemiology of cardiovascular disease in the UK 2014. Heart 101: 1182-1189, 2015.

65. Vasan Ramachandran S and Peter WF Wilson: Epidemiology and causes of heartfailure. Wolters Kluwer Health Clinical Solutions: 2015. 